\title{
Community proteogenomics highlights microbial strain-variant protein expression within activated sludge performing enhanced biological phosphorus removal
}

\author{
Paul Wilmes ${ }^{1}$, Anders F Andersson ${ }^{1}$, Mark G Lefsrud ${ }^{2}$, Margaret Wexler ${ }^{3}$, Manesh Shah ${ }^{4}$, \\ Bing Zhang ${ }^{5}$, Robert L Hettich ${ }^{6}$, Philip L Bond ${ }^{7}$, Nathan C VerBerkmoes ${ }^{6}$ \\ and Jillian F Banfield ${ }^{1,8}$ \\ ${ }^{1}$ Department of Earth and Planetary Sciences, University of California at Berkeley, Berkeley, USA; \\ ${ }^{2}$ Bioresource Engineering, McGill University, Ste-Anne-de-Bellevue, Canada; ${ }^{3}$ School of Biological Sciences, \\ University of East Anglia, Norwich, UK; ${ }^{4}$ Life Sciences Division, Oak Ridge National Laboratory, Oak Ridge, \\ USA; ${ }^{5}$ Department of Biomedical Informatics, Vanderbilt University Medical Center, Nashville, USA; \\ ${ }^{6}$ Chemical Sciences Division, Oak Ridge National Laboratory, Oak Ridge, USA; ${ }^{7}$ Advanced Wastewater \\ Management Center, University of Queensland, St Lucia, Queensland, Australia and ${ }^{8}$ Department of \\ Environmental Science, Policy, and Management, University of California at Berkeley, Berkeley, USA
}

\begin{abstract}
Enhanced biological phosphorus removal (EBPR) selects for polyphosphate accumulating microorganisms to achieve phosphate removal from wastewater. We used high-resolution community proteomics to identify key metabolic pathways in 'Candidatus Accumulibacter phosphatis' (A. phosphatis)-mediated EBPR and to evaluate the contributions of co-existing strains within the dominant population. Overall, 702 proteins from the $A$. phosphatis population were identified. Results highlight the importance of denitrification, fatty acid cycling and the glyoxylate bypass in EBPR. Strong similarity in protein profiles under anaerobic and aerobic conditions was uncovered (only $3 \%$ of $A$. phosphatis-associated proteins exhibited statistically significant abundance differences). By comprehensive genome-wide alignment of 13930 orthologous proteins, we uncovered substantial differences in protein abundance for enzyme variants involved in both core-metabolism and EBPR-specific pathways among the A. phosphatis population. These findings suggest an essential role for genetic diversity in maintaining the stable performance of EBPR systems and, hence, demonstrate the power of integrated cultivation-independent genomics and proteomics for the analysis of complex biotechnological systems.

The ISME Journal (2008) 2, 853-864; doi:10.1038/ismej.2008.38; published online 1 May 2008

Subject Category: integrated genomics and post-genomics approaches in microbial ecology

Keywords: 'Candidatus Accumulibacter phosphatis'; community proteogenomics; enhanced biological phosphorus removal; metaproteomics; proteomics; strain variation
\end{abstract}

\section{Introduction}

Inorganic phosphate $\left(\mathrm{P}_{\mathrm{i}}\right)$ is a principal limiting nutrient in freshwater ecosystems. High nutrient

Correspondence: JF Banfield, Department of Earth and Planetary Sciences, Department of Environmental Science, Policy, and Management, University of California at Berkeley, Berkeley, CA 94720, USA.

E-mail: jbanfield@berkeley.edu and

PL Bond, Advanced Wastewater Management Center, University of Queensland, St Lucia, Queensland 4072, Australia.

E-mail: phil.bond@uq.edu.au

Received 18 January 2008; revised 25 March 2008; accepted 25 March 2008; published online 1 May 2008 loads, mainly from anthropogenic sources, impact on the quality of surface waters and may promote the deleterious effect of eutrophication (Correll, 1998).

Rapid human population growth and global climate change are placing considerable pressures on the global water cycle (United Nations World Water Assessment Programme, 2006). Aquatic $\mathrm{P}_{\mathrm{i}}$ levels can be reduced through biological wastewater treatment. Activated sludge undergoing alternating anaerobic and aerobic regimes favors the growth of polyphosphate accumulating microorganisms that enable enhanced biological phosphorus removal (EBPR; Seviour et al., 2003). Continuous removal 
of defined amounts of polyphosphate-rich biomass facilitates $P_{i}$ removal and the treated supernatant can be safely discharged into surface waters.

EBPR installations are used extensively throughout the world because they are considered more cost-effective and sustainable compared to chemical precipitation (Blackall et al., 2002). Although EBPR has been empirically optimized since its fortuitous discovery in the 1950s (Srinath et al., 1959), detailed knowledge of the microbiology and biochemistry is required to improve operational stability.

Since its inception, basic research on EBPR has been plagued by enrichment bias. Several potential polyphosphate accumulating organism (PAO) isolates (predominantly Acinetobacter spp.) were obtained from EBPR biomass but their phenotypes were inconsistent with the biochemical transformations observed in full- and laboratory-scale systems (Blackall et al., 2002; Seviour et al., 2003). With the advent of molecular tools (16S rRNA sequences), a dominant phylotype belonging to the order Rhodocyclales was identified within EBPR-activated sludge (Bond et al., 1995; Hesselmann et al., 1999; Crocetti et al., 2000). Putatively named 'Candidatus Accumulibacter phosphatis' (A. phosphatis; Hesselmann et al., 1999), it has since been repeatedly detected in activated sludge in various locations on Earth (Blackall et al., 2002).

Although the identity of $A$. phosphatis as a major PAO has been established, details regarding its metabolism have proven controversial (Seviour et al., 2003). Even the recent landmark paper by García Martín et al. (2006), describing the genetic inventory of dominant $A$. phosphatis populations, has not abated discussions regarding its metabolic potential. Causes célèbres include the ability of $A$. phosphatis to denitrify (Kong et al., 2004; García Martín et al., 2006), the nature of its glycolytic pathway (Hesselmann et al., 2000; García Martín et al., 2006) and how it generates reducing equivalents during the anaerobic phase (Mino et al., 1998; García Martín et al., 2006). The ability to detect proteins involved in candidate pathways should help shed light on these unresolved issues.

Proteomics employing liquid chromatography (LC) coupled with tandem mass-spectrometry (MS) can provide comprehensive high-resolution analysis of the protein complement of natural microbial communities (Ram et al., 2005; Wilmes and Bond, 2006a; Lo et al., 2007). So far, comprehensive community proteogenomic analyses have provided unprecedented insight into the structure and function of microbial biofilms inhabiting warm, extremely acidic and metal-rich solutions within the Richmond Mine at Iron Mountain, California, USA (Ram et al., 2005; Lo et al., 2007). These biofilms are ideally suited for the development of community genomic and proteomic methodologies due to their limited species richness and abundant biomass. However, the application of proteogenomic techniques to more complex microbial ecosystems pre- sents additional challenges. Here we apply shotgun proteomics to a complex activated sludge community cultured in a laboratory-scale sequencing batch reactor (SBR) operated in the United Kingdom (UK), enriched for A. phosphatis and performing EBPR at high $\mathrm{P}_{\mathrm{i}}$ influent levels. Metagenomic sequences obtained from laboratory-grown sludges derived from wastewater treatment plants in the United States (US) and in Australia (OZ; García Martín et al., 2006) were employed to identify abundant proteins. The identified proteins were interpreted within the context of current EBPR metabolic models. Importantly, very high mass accuracy data were obtained using a linear ion trap (LTQ)-Orbitrap mass spectrometer. This allowed confident identification of proteins derived from distinct strains within the $A$. phosphatis population. Differences in the abundances of protein variants associated with different $A$. phosphatis subpopulations may be a reflection of functional partitioning within the population and highlight the apparent importance of genetic diversity in maintaining stable process performance.

\section{Materials and methods}

Laboratory-scale SBR

A laboratory-scale SBR with alternating anaerobic/ aerobic phases was operated as previously reported (Wilmes and Bond, 2004, 2006b). The reactor was initially seeded with activated sludge from the Whitlingham WWTP, Norwich, UK. Stable EBPR was obtained with complete removal of $55 \mathrm{mgl}^{-1}$ of $\mathrm{P}_{\mathrm{i}}$ from the synthetic wastewater feed with acetate as the main carbon source (Wilmes et al., 2008).

\section{Clone library construction and sequence analysis}

DNA was extracted as described earlier (Bond et al., 1995). PCR primers specific for 'Candidatus Accumulibacter' polyphosphate kinase 1 (Acc-ppk1-254f and Acc-ppk1-1367r) were used for amplification as previously described (He et al., 2007). Primers specific for sludge malate synthase were designed using the metagenomic sequences and were as follows: MS3F (5'-ATTCCGATCAAGAACGATCC-3') and MS3R (5'-CTCAGCGACATCTTGTCGAA-3'). Clone libraries were prepared using the TOPO TA Cloning kit (Invitrogen, Paisley, UK). Nine clones were analyzed per gene, grouped by restriction enzyme analysis and representatives sequenced. Evolutionary analyses of sequence data were performed by distance methods using ARB (Ludwig et al., 2004). DNA base sequence was converted to amino acid sequence, aligned and phylogenetic trees were assembled by neighbor joining and maximum likelihood analyses. The GenBank accession numbers for the nucleotide sequences determined in this study are EU563082 to EU563085. 


\section{Proteomics}

Proteins were extracted from the SBR biomass at the end of the anaerobic and aerobic phases as described earlier (Wilmes and Bond, 2004, 2006b). Trichloroacetic acid precipitated protein pellets were resuspended in $6 \mathrm{M}$ Guanidine/10 mM DTT for denaturation and reduction, and then digested into peptides with sequencing grade trypsin (Promega, Madison, WI, USA). Samples were de-salted, filtered and concentrated. The anaerobic and aerobic samples were analyzed via 24-h, 12-step 2-D LC (SCX-RP)-MS/MS runs on an LTQ and a hybrid LTQOrbitrap with technical duplication (Ram et al., 2005; Lo et al., 2007). Both instruments were operated in data-dependent MS/MS mode. For the LTQ-Orbitrap, full scans were acquired at 30000 resolution in the Orbitrap, while MS/MS scans were acquired in the LTQ. Separate and combined database searches were preformed with SEQUEST (Eng et al., 1994) against the three available sludge metagenomic databases (García Martín et al., 2006; Supplementary Table 1). Detailed information on the metagenomic sequences was retrieved using the integrated microbial genomes with microbiome samples (IMG/M) system (Markowitz et al., 2006). In addition to the metagenomic protein sequences, common contaminants (trypsin, keratin, etc.) were also included in the search database. MS data were filtered with DTASelect (Tabb et al., 2002) at the peptide level [Xcorrs of at least $1.8(+1), 2.5(+2)$, $3.5(+3)]$. For positive protein identification, a minimum of two fully tryptic peptides was required per protein in at least one replicate run.

False-positive levels were estimated by reverse database searching and were about $1 \%$ for both instruments (deltCN filter of 0.08). High mass accuracy monoisotopic peaks were extracted from the LTQ-Orbitrap full scans and compared to theoretical monoisotopic masses for all identified peptides. Overall, 70\% of proteins were identified using both instruments.

To test the uniqueness of identifications against more extended database space, a large database was created and MS data from two runs were searched against it. This database contained: the Phrap assembly of the US sludge (USP), AMD metagenome (12 148 proteins), 249 microbial isolates from IMG (924816 proteins). From this search, 90\% of the unique peptides matched the USP database and only $10 \%$ matched with the other database entries. All datasets, databases and supplementary files (spreadsheets in.xls format) can be viewed at http:// compbio.ornl.gov/ebpr_sludge.

Protein abundances were determined from sequence coverage (percentage of protein covered by detected peptides), total spectral count (number of spectra detected per protein), unique peptide count (number of times any unique peptide is detected from a protein) and normalized spectral abundance factor (Florens et al., 2006). We compared the differences in protein abundances between the anaerobic and aerobic phases using a Poisson regression model with total spectral count as the outcome and phase as the main independent variable. In addition, we included instrument as an independent variable to adjust for a potential confounding instrument effect. As we were comparing differential expression for 4688 proteins (combined database), $P$-values generated by the model were further adjusted using the Benjamini and Hochberg correction to account for multiple comparisons (Benjamini and Hochberg, 1995). An adjusted $P$-value of 0.10 (that is $10 \%$ False Discovery Rate) was used to select proteins differentially expressed between the anaerobic and aerobic phases.

\section{Tetranucleotide frequency calculation and self- organizing maps}

A Perl script was written to calculate tetranucleotide frequencies of the US sludge's Jazz assembly scaffold sequences, serving as input for self-organizing map (SOM) training. We employed the Databionics ESOM program package (Ultsch and Moerchen, 2005) for training and visualizing of self-organizing maps (Kohonen, 1990). To reduce coding strand biases, frequencies of pairs of reverse complementary tetranucleotides were summed (Abe et al., 2005). A $328 \times 100$ torroid SOM was trained on scaffolds $\geqslant 2000 \mathrm{bp}$. Scaffolds $\geqslant 10000 \mathrm{bp}$ were fragmented into subsequences by splitting after each $5 \mathrm{~kb}$, such that the last subsequence contained $\geqslant 5000$ and $<10000 \mathrm{~kb}$ of sequence. Weights were initialized by principal component analysis, the order of the input data was randomized and 40 training epochs of $15 \%$-batch training were performed. Onto the resulting map, the tetranucleotide frequencies of all scaffolds $\geqslant 1000 \mathrm{bp}$ were projected. Scaffolds $\geqslant 10000 \mathrm{bp}$ were fragmented as above. Scaffold fragments were highlighted according to previous binning information (García Martín et al., 2006; McHardy et al., 2007). For this, information from the different binning methods (US/OZ overlap, PhyloPythia $>85 \%$ confidence and PhyloPythia $<85 \%$ confidence) was concatenated according to highest taxonomic affiliation. A scaffold sequence fragment was considered expressed if it overlapped with $>50 \%$ of an open reading frame (ORF) that was detected in any of the mass-spectrometry runs. The background was colored according to the U-matrix (Ultsch and Moerchen, 2005).

\section{Ortholog alignment}

A programme was written in Perl for aligning contigs and scaffolds against a predefined set of backbone scaffolds, based on sequence similarity of the encoded proteins. Protein sequence similarity was deduced by BLASTP (Altschul et al., 1997); the sequence identity for a pair of proteins was defined 
as the number of identical amino acids divided by the length of the smallest protein. Starting with the largest backbone scaffold and progressing in decremental order, all previously non-aligned, non-backbone contigs and scaffolds were aligned fulfilling the following criteria: (a) At least $50 \%$ of the proteins in the non-backbone contig or scaffold were at least $90 \%$ identical with proteins in the backbone scaffold, (b) The matching proteins were syntenous in the aligned contigs and/or scaffolds, allowing a maximum of $10 \mathrm{ORF}$ insertions in either of the contigs or scaffolds, between any two matching proteins.

The resulting alignment file can be viewed in a spreadsheet format with the backbone scaffolds displayed in the first column, with one IMG/M gene object identifier per row and aligned contigs and scaffolds in succeeding columns. The Perl programme is available from the authors upon request.

\section{Results}

SBR performance and community structure

A laboratory-scale SBR was successfully operated for complete removal of around $55 \mathrm{mgl}^{-1}$ of $\mathrm{P}_{\mathrm{i}}$ from synthetic wastewater feed (Wilmes et al., 2008).

Fluorescence in situ hybridization employing $16 \mathrm{~S}$ rRNA oligonucleotide probes revealed dominance of A. phosphatis $(69 \%$ of bacterial cells, s.d. 10.8; Wilmes et al., 2008). As the $16 \mathrm{~S}$ rRNA locus is not ideally suited for discriminating between closely related organisms, we sequenced fragments of the polyphosphate kinase 1 (ppk1) gene, a more sensitive phylogenetic marker for distinguishing Accumulibacter species and $A$. phosphatis strains ( $\mathrm{He}$ et al., 2007). We retrieved three distinct ppk1 gene sequences from the UK sludge (representing nine clones) and compared them to previously reported ppk1 sequences from EBPR-activated sludges (Supplementary Figure 1 online). This revealed phylotypes in the UK sludge that were distinct from the US and OZ phylotypes. Furthermore, the UK sequences clustered into two specific clades (I and IID), suggesting greater phylogenetic diversity in the UK sludge compared to the US and OZ sludges.

\section{Protein identifications}

Proteins were extracted from the UK sludge at the end of the anaerobic $(t=120 \mathrm{~min})$ and the aerobic phases $(t=330 \mathrm{~min})$, proteolytically digested with trypsin and analyzed in duplicate via two-dimensional nano-LC followed by MS/MS analysis using either an LTQ or a hybrid LTQ-Orbitrap mass spectrometer. Peptide tandem mass spectra were matched in silico to predicted peptides and, thus, proteins in the sludge metagenomic databases (García Martín et al., 2006; Supplementary Table 1 online). Three distinct metagenomic sequence databases were used for protein identification: USJazz
(Jazz assembly of the US sludge; USJ), USPhrap (US Phrap assembly; USP) and OZPhrap (OZ Phrap assembly; OZP). For positive protein identification, a minimum of two peptides were required per protein in at least one replicate run (Ram et al., 2005). The MS data were searched separately against the metagenomic databases and against a combined database (Supplementary Table 2). A total of 5029 proteins were identified using both instruments and database combinations (Supplementary Table 3). However, this number is inflated due to database redundancy (mainly between USJ and USP). When searching the mass spectra solely against the OZP and USP databases (of similar size), 916 and 980 (LTQ and LTQ-Orbitrap) versus 1857 and 1728 nonredundant positive identifications were obtained, respectively. The results suggest that the UK sludge contained genes present in both the OZ and US sludges. However, based on protein identifications, the genetic composition of the UK sludge appears more similar to the US sludge than the OZ sludge.

Assignment of identified proteins to organisms Overall, $36 \%$ of identified proteins were encoded by genes located on genomic contigs or scaffolds assigned to A. phosphatis (García Martín et al., 2006; McHardy et al., 2007; Figure 1a). In addition, several identified proteins were inferred to derive from other Accumulibacter species or other $\beta$ Proteobacteria (2 and $3 \%$, respectively). Numerous proteins affiliated with $\gamma$-Proteobacteria were also identified $(3 \%)$.

In all, $56 \%$ of identified proteins were encoded on contigs and scaffolds whose organismal affiliation was uncertain based on the previous metagenomic analysis (García Martín et al., 2006; McHardy et al., 2007). Many of these proteins were found to be highly abundant, based on protein sequence coverages, total spectral counts and normalized spectral abundance factors (NSAFs; Florens et al., 2006) and likely derive from other $A$. phosphatis strains, other Accumulibacter species, or active members of the flanking populations.

In total, $14 \%$ of all detected proteins were of unknown function, $18 \%$ of which exhibited uncharacterized conserved domains. Interestingly, the largest number of assigned mass spectra was obtained for an unclassified protein of unknown function encoded on a USP contig (IMG/M gene object identifier: 2000289750). This protein has a conserved domain homologous to zinc peptidases found in several Proteobacteria, Chlorobiaceae and Rhodopirellula baltica and was more abundant in the aerobic protein extract. The specific role of this protein in the context of EBPR has yet to be determined.

To constrain the affiliation of sequences related to, but distinct from, the composite $A$. phosphatis genome (García Martín et al., 2006), we calculated tetranucleotide frequencies for all USJ scaffolds and 

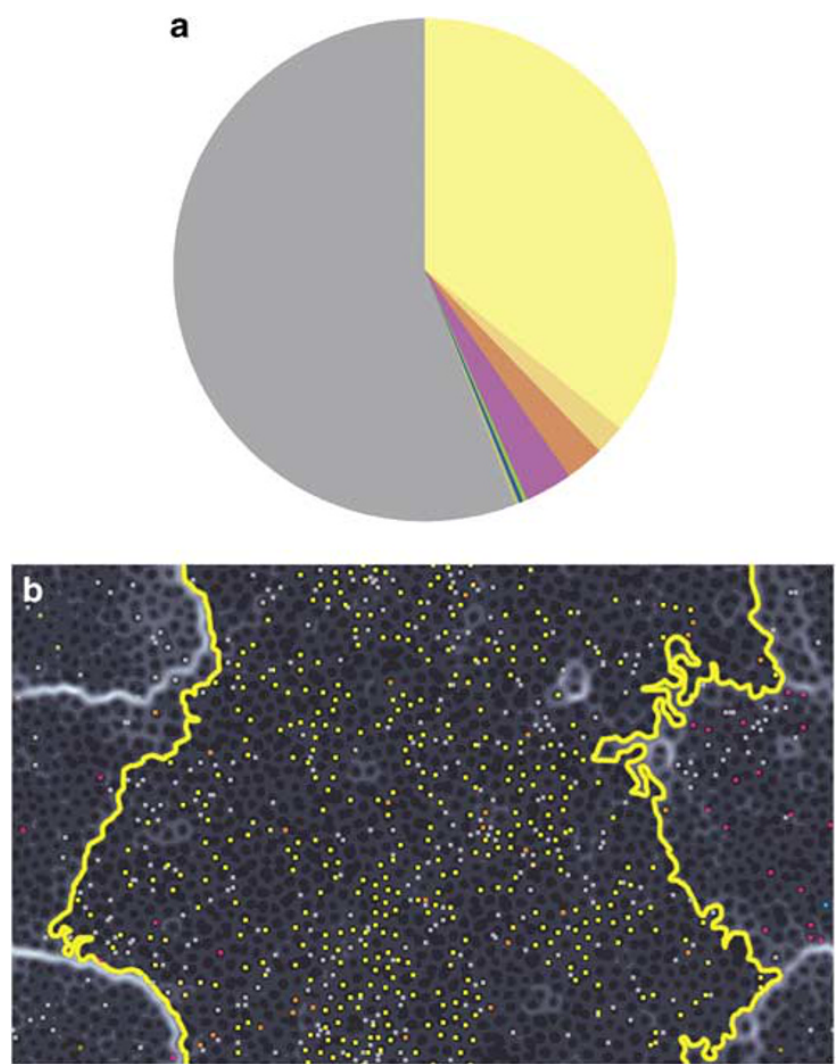

Figure 1 Taxonomic affiliation of identified proteins. (a) Proportions of all identified proteins that belong to taxonomic groups based on concatenated genomic contig and scaffold binning (García Martín et al., 2006; McHardy et al., 2007). (b) Self-organizing map based on tetranucleotide frequency of USJ scaffold fragments with genomic fragments encoding identified proteins highlighted according to taxonomic groups (the $A$. phosphatis fragment cluster is delimited by a yellow line). Information in both panels is color-coded according to the concatenated binning information: A. phosphatis, Accumulibacter, Betaproteobacteria, Gammaproteobacteria, Thiothrix, Actinobacteria, Alphaproteobacteria, Sphingobacteria, D Deinococci, D Deltaproteobacteria, Clostridia, Planctomycetacia, Spirochaetes, Methanomicrobia, Chromadorea, Insecta, Mollicutes, unclassified.

visualized the results in two-dimensional space using a self-organizing map (SOM; Kohonen, 1990; Abe et al., 2005). To identify and evaluate the SOM clusters, we overlaid the corresponding sludge binning information (Supplementary Figure 2a). A notable feature is the large fraction of unclassified scaffold fragments that cluster with $A$. phosphatis, Accumulibacter or $\beta$-Proteobacteria (64\% of scaffold fragments) and, hence, these likely derive from closely related organisms.

We overlaid protein expression data on the SOM. A total of $89 \%$ of the genomic fragments encoding identified proteins fall within the A. phosphatis region (Figure 1b and Supplementary Figure 2b), and $41 \%$ of these were encoded on previously unclassified scaffolds. These genomic fragments encoding identified proteins of possible $A$. phosphatis strains may have marked functional significance in the UK sludge.
Analysis of A. phosphatis strain variation

Strain variation among closely related organisms in complex microbial communities may result from single nucleotide polymorphisms, genome rearrangements, as well as gene insertions and deletions. These events can fragment genomic assemblies. Resulting small contigs and scaffolds may be placed in genome context by mapping them against the assembled composite $A$. phosphatis sequence (García Martín et al., 2006) using syntenous and shared genes. We developed an automated approach for aligning variant contigs and scaffolds that required $50 \%$ of the ORFs on a fragment to share $>90 \%$ amino acid sequence identity with the composite backbone genome and allowing a maximum of $10 \mathrm{ORF}$ insertions (Supplementary Table 4). The 50\% aligned ORF criterion was empirically determined to account for differences in genetic variation among neighboring genes, and the $90 \%$ amino acid identity cutoff was chosen so that orthologous proteins would be identifiable by both unique and non-unique peptides in most cases (Denef et al., 2007). Unique peptides are specific to a given protein in the database, whereas non-unique peptides may be derived from multiple proteins. Gaps in the alignment of orthologous contigs and scaffolds highlight the importance of gene insertions and deletions within the $A$. phosphatis population (Supplementary Table 4).

\section{A. phosphatis protein expression}

The composite A. phosphatis genomic fragments from USJ and aligned contigs and scaffolds from USJ, USP and OZP were used to interpret $A$. phosphatis protein expression. Overall, 559 proteins were identified using the composite genome backbone (MS data searched against separate databases; average protein coverage: 13\%). This represents around $10 \%$ of the predicted $A$. phosphatis genes (and more than half of all non-redundant proteins identified using USJ). 1534 proteins with orthologs on the backbone were identified on aligned contigs and scaffolds, representing a non-redundant count of 556 proteins. Among these, 78 were strainspecific variants identified based on peptides specific to proteins encoded by aligned contigs and scaffolds and, hence, not identified using the backbone sequences. In addition, 1338 proteins encoded by aligned contigs and scaffolds but not by the backbone sequence were recruited and 68 of these were identified by proteomics. These aligned proteins are encoded by inserted genes within the variant subpopulations.

Many orthologs present on the backbone and aligned contigs and scaffolds were identified based on unique and non-unique peptides. Overall, proteins encoded by aligned scaffolds and contigs were found to be more abundant than the backbone variants in 414 cases (based on total spectral counts; Supplementary Figure 3 and Supplementary Table 5). 
Proteomics-enabled metabolic insights

The abundances of proteins identified in the anaerobic and aerobic phases were very similar for both instruments (Supplementary Figure 4). Four percent of all identified proteins exhibited statistically significant differences in abundance between the anaerobic and aerobic phases (see Methods; Supplementary Table 6). Only 3\% of identified proteins from the $A$. phosphatis population and $4 \%$ of proteins not associated with the $A$. phosphatis population showed different abundance levels between the two phases. These results were unexpected, given distinct differences in chemical transformations performed under each condition. However, some differentially expressed proteins could be directly linked to the metabolic transformations characteristic of EBPR, including poly(3hydroxyalkanoate) synthase [anaerobic polyhydroxyalkanoate (PHA) synthesis], glycosidase and glucan phosphorylase (anaerobic glycogenolysis), glyceraldehyde-3-phosphate dehydrogenase (anaerobic glycolysis) and glycogen synthase and 1,4-alpha-glucan branching enzyme (aerobic glycogen synthesis).

Protein abundance information was placed into metabolic context and the extent to which proteins may play dual roles under anaerobic and aerobic conditions was evaluated. Proteins were assigned to processes involved in $\mathrm{P}_{\mathrm{i}}$ transformations, PHA cycling, fatty acid cycling, tricarboxylic acid (TCA)/ glyoxylate cycle, carbohydrate transformations, energy production and conversion, denitrification and exopolysaccharide (EPS) synthesis (Figure 2).

Many of the proteins involved in the metabolic pathways exhibited sequence variation compared to the A. phosphatis composite genome. Notably, proteins encoded by aligned genome fragments and involved in key metabolic transformations were found to be more abundant than proteins encoded by the backbone reference sequence (Figure 3a, Table 1, Supplementary Figure 3 and Supplementary Table 5). In particular, a large proportion of variant proteins involved in fatty acid metabolism, the TCA/glyoxylate cycle and sugar metabolism exceeded backbone proteins in terms of abundance (Table 1 and Supplementary Figure 3). For example, the proteomic results indicated that the malate synthase protein variant (USP_MALS_1) encoded by an aligned contig was more abundant than that encoded by the backbone (Figure 3a). We confirmed this deduction using the dominant malate synthase gene sequence amplified from our bioreactor (Figure 3b).

\section{Discussion}

Using proteogenomics, we identified 702 proteins from the $A$. phosphatis population present in the UK sludge. This provided sufficient information to conduct a detailed analysis of metabolic activity during EBPR. Apart from proteins affiliated with $A$. phosphatis, numerous proteins associated with $\gamma$ Proteobacteria were identified. The presence of $\gamma$ Proteobacteria was previously reported in failing EBPR systems (Crocetti et al., 2002), and they have been shown to actively compete with PAOs over carbon substrates (Kong et al., 2005). Indeed, the abundant $\gamma$-proteobacterial proteins identified suggest a metabolism analogous to A. phosphatis' carbon metabolism.

A. phosphatis and closely related organisms are cycled through alternating anaerobic and aerobic phases, both characterized by specific metabolic transformations. During the anaerobic phase, polyphosphate (polyP) and glycogen are degraded, and PHAs are synthesized from short chain volatile fatty acids. This process is reversed during the aerobic phase with the replenishment of both polyP and glycogen in conjunction with the hydrolysis of PHAs. Owing to the distinct metabolic transformations apparent in the two contrasting phases, protein expression differences were anticipated between both conditions. However, protein abundances were similar for the vast majority of metabolic enzymes, as highlighted in the metabolic model (Figure 2). Only $3 \%$ of identified proteins from the $A$. phosphatis population versus $4 \%$ of proteins not associated with the $A$. phosphatis population exhibited expression differences between the two phases. This may result in part from carryover of proteins from the anaerobic ( $115 \mathrm{~min}$ long) to the aerobic (210 $\mathrm{min}$ ) phase and vice versa. However, an interesting possibility is that the majority of enzymes are able to catalyze reactions in both forward and reverse directions. For example, in E. coli, the enzymes involved in glycolysis and glucogenesis are shared except for the two key enzymes that regulate the directionality of the carbon flow, that is phosphofructokinase (glycolysis) and fructose-1,6-biphosphate phosphatase (gluconeogenesis) and these are regulated allosterically by intracellular levels of ADP and AMP, respectively (White, 2007). Similarly, allosteric regulation of key metabolic steps in A. phosphatis would enable rapid switching in response to alternating anaerobic and aerobic conditions and provide a bioenergetic advantage to the

Figure 2 Proposed model for EBPR-specific metabolism carried out by the A. phosphatis population during the (a) anaerobic and (b) aerobic phases. Proteins involved in $\mathrm{P}_{\mathrm{i}}$ transformations, polyhydroxyalkanoate (PHA) cycling, fatty acid cycling, tricarboxylic acid/ glyoxylate cycle, carbohydrate transformations, energy production and conversion, dentrification and exopolysaccharide synthesis are highlighted according to their relative abundances. Full arrows indicate the presence of the coding gene on the A. phosphatis composite genome, dashed arrows refer to orthologous identified proteins. For more information on identified proteins, please see Supplementary Table 7 online. 


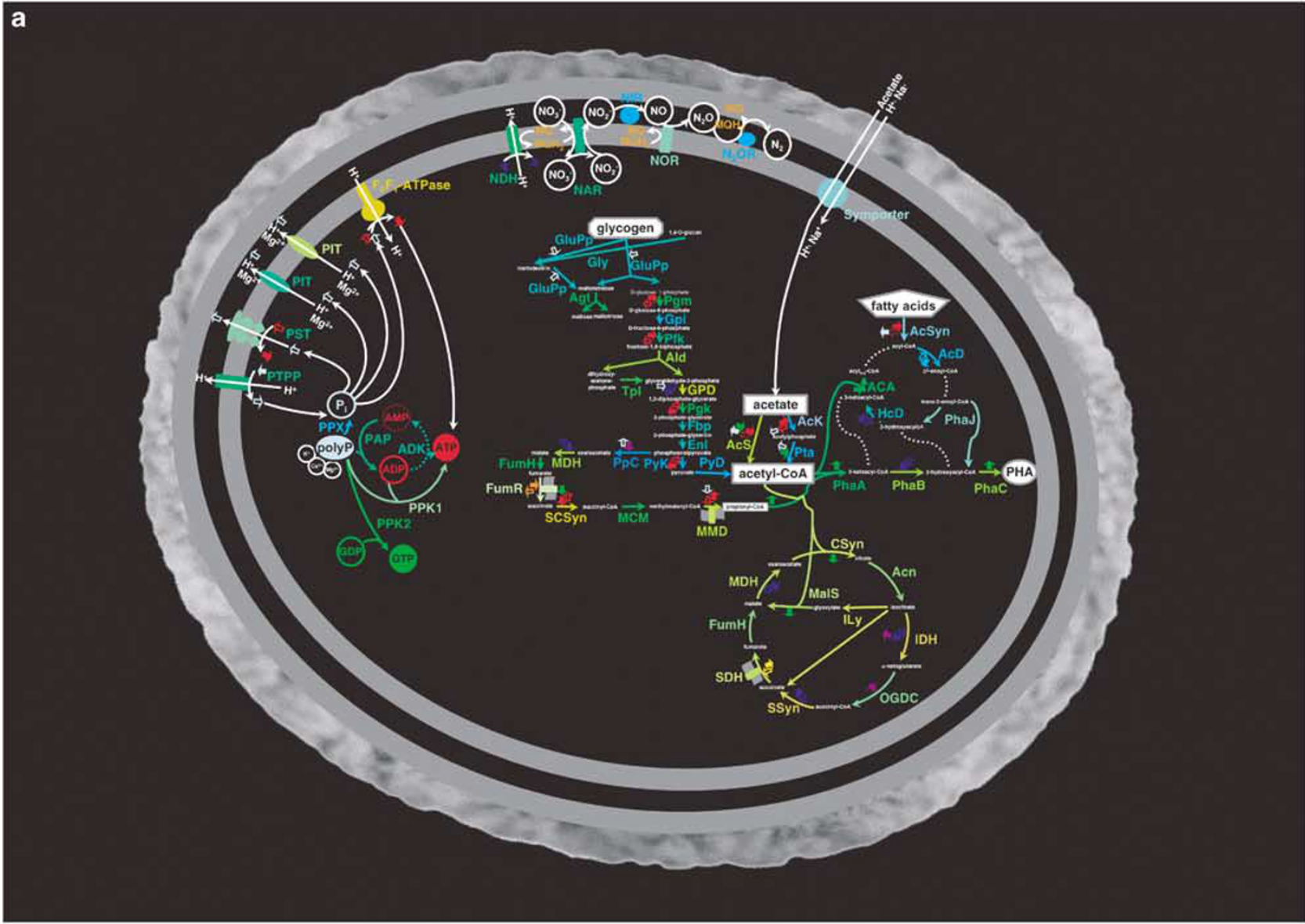

b

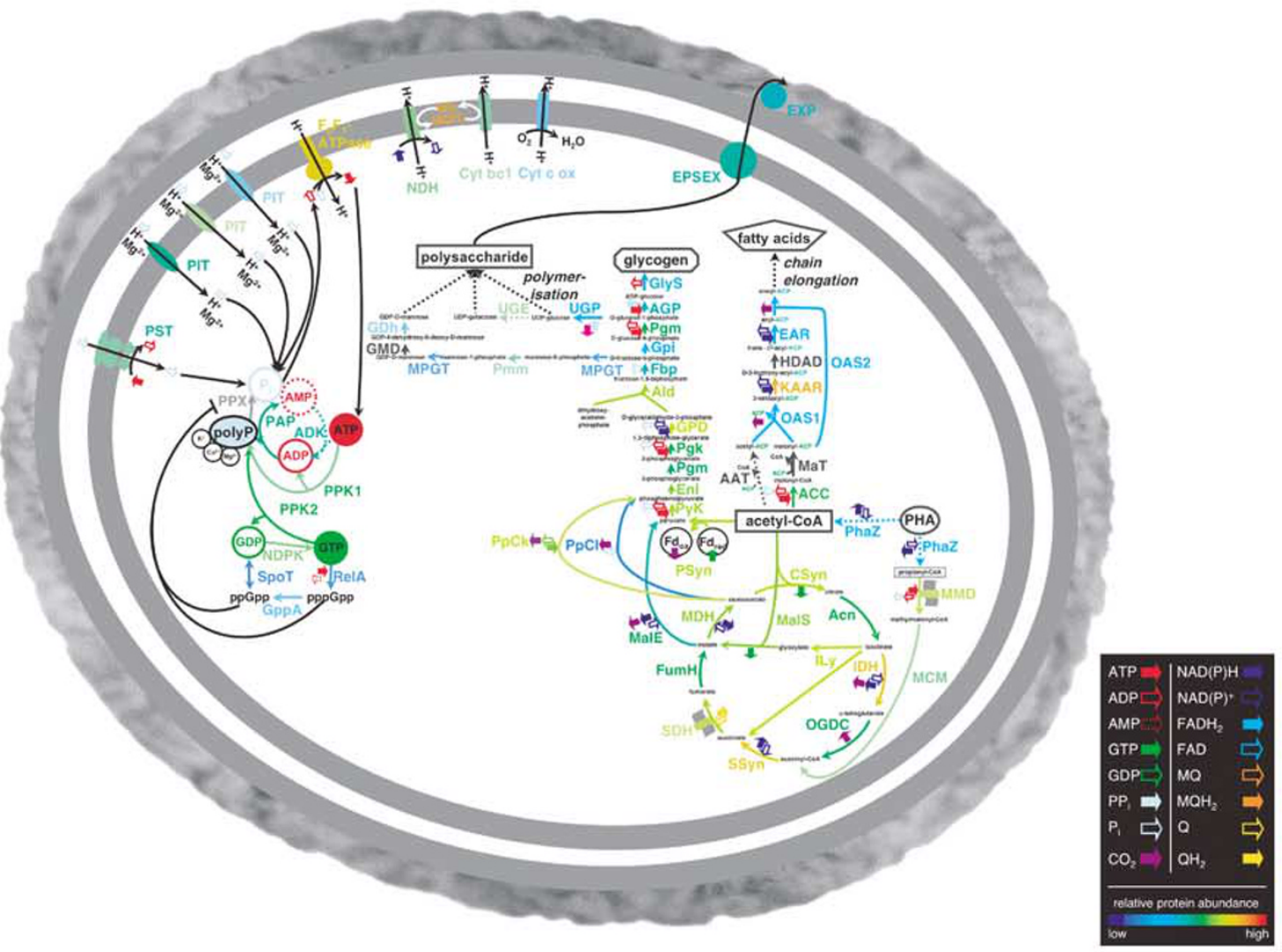


A. phosphatis population, since protein synthesis and degradation are costly processes. A similar suggestion was made in a previous low-resolution

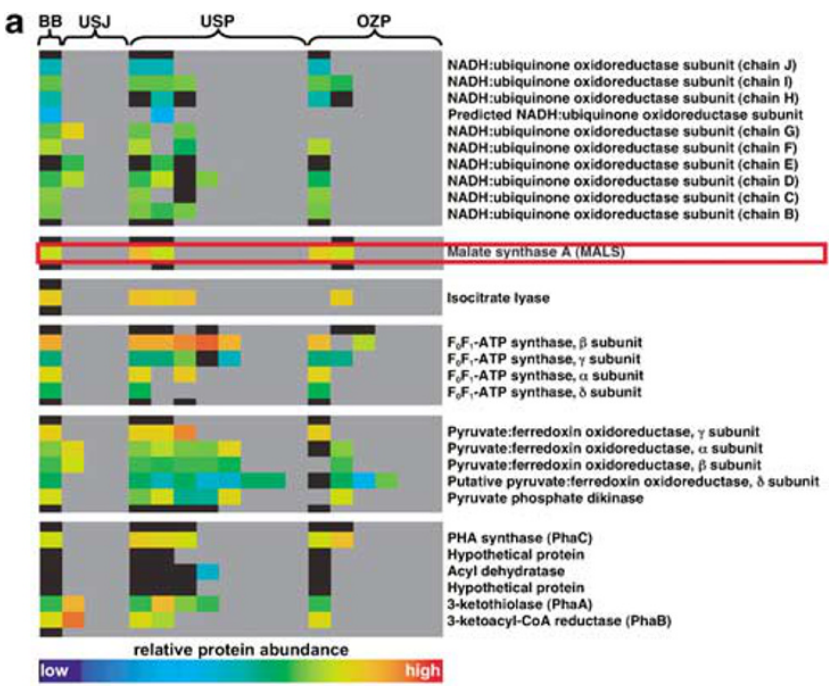

\section{b}

WJGRSALIPIKRDPEAMEKALAGIRHDKTRDANDGFDGGVAHPGLVGIAMEgFOKVIOE aggaagcacaaagcggagageggaccgaacggaggtgggtggcegeggaggggtcageg acacaaactcgtgaaacgacaagtagggtcacgttgtccaataatt

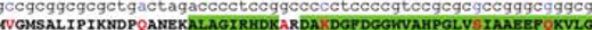
aggaagcacaaagccgagageggacegageggaggtgggtgggecgegtaggggteagcgg
ttgtgcttctaaacacaaactcgtgaaacgacaagtagggtcacgttctccaataattga

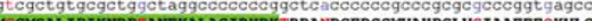
Tyag aggaagcacaaagoggagagcggaccgaa cggaggtgggtggocgeggaggggtcagcgg gecgeggegeget gactagaccectecggececetccccgtccgcgcgecgggegggcg aggaagcacaaagcggagagcggaccgaacggaggtgggtggccgegtaggggtcagcg
tggtgct tctaaacteaaactcgtgaaacgacaagtagggtcacgttctccaataattga geggtggtgegttggecgggececececggeccececetegt gegagegeceggteggea aggaagcacaaagcggagagcggaccgaacggaggtgggtggccgcggaggggtcagcgg gccgeggegeget gacta gaccoctec ggeccectccccgtcecgegegecgggegggoc

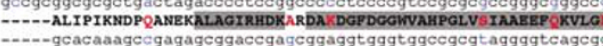
----cttctaaacacaaactcgtgaaacgacaagtagggtcacgttctccaataatt ----cttctaaacacaaactcgtgaaacgacaagtagggtcacgttctccaataattga

B8_MaLs

USP_MaLs_1

USP_MaLs_2

OZP_MaLs_1

OZP_MaLs_2

ux_MaLs

RPHQWEKORDETFTAADFLMFQPSOPITEAGVRMUINVGIHYLGSWLAGMGCVPIHMLLE ccactgaccggatagggtcatcctccaaggggcaaaaggactcgatcggagtgcacacag

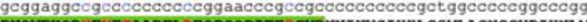

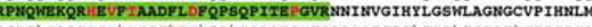

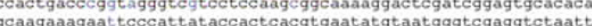

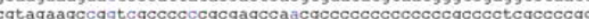
RPWOWEKORDETFTAADPLAPQPSOPITEAGVRNMTNVGIHYLGSWLAGNGCVPIHMLVE ccactgaceggatagggteatcetceaaggggeaaanggactegateggagtgcacaca gcaagaaagaactcccattataccactcacgtgaatatgtaatgggtcgaggtctaatt Rgcggaggccgccccccccccggaacccgccgccccccccccgctggcccccggcccgga

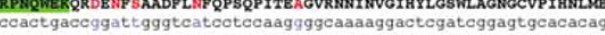
gcaagaaagaaatcccattataccactcacgtgaatatgtaatgggtegaggtctaat

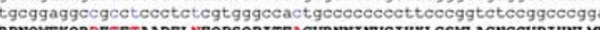
RPNQWEKQRDETFTAADFLMFQPSQPTTEAGVRMHINVGIHYLGSWLAGMCCVPIHNLLG ccactgaccggatagggtcatcctccaaggggcaaaaggactcgatcggagtgcacacag
gcaagaaagaactcccattataccactcacgtgaatatgtaatgggtegaggtctaatt gcaagaaagaacteccatta taocactcaegtgaatatgtaatgggtegaggtctaatta Rgeggaggecgecceccecceggaagecgecgececceccegctggececggecegga ccactgacccggtagggtchtcctccaagcggcaaaggactcgatcggagtgcacacag

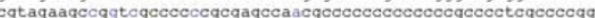

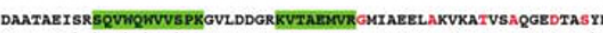
gggaggatetcgtct ggtcaggegggeagaggagegaagggegagagagagegggagat ccocotog ccgacgcgcggchaggtgagtgccttcgeccgggcc gccgagcaggaggccgcaccctcC gggaggatetcgtetggteaggegggeagaggagegaagggegagagggagegggagatg cgaccgcgcggcgagggcgactcct tgcqcccalggccgtcogaggggggaccagtacgccce DAATAE ISR SQVWQWVVSPRGVLDDGRKVTAENVR GMIAEELAKVKATVSAQGEDTASYD gggaggatctcgtct ggtcaggcgggcagaggagcgaagggcgagagagagcgggagatg cegacgegeatgagteceagttaaggatecattggttcaatcatacetgcagaacega

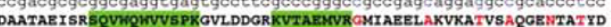
gggaggatctcgtctggtcaggcgggcagaggagcgaagggcgagagagaacggaagatg
accccatcgcatgagttccagttaaggatccattggttcaatcatacctgcagaaccoaa cagggacgtgggggggtegaacecegegecegggeagetaageggatececgagegecct DAATAE ISRSQVWRWVVSPKGVLDDGRKVTAERVR GMCAEELAKVKATVSTQGEDTASYD

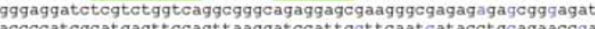
cceccatcgeatgagteccagttaaggatccat tggttcaatcatacctgcagaaccya

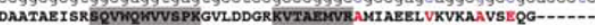
gggaggatctcgtctggtcaggegggcagaggagegaagggegagagggageg-----cgaccgcgcggegagggcgactcctgcgcccaggecgtcgaggggggaccag

ozp_ras__2 2

uK_mats proteomic study (Wilmes and Bond, 2006b). Alternatively, similarities in protein profiles between the two alternating growth conditions may indicate a role for post-translational modifications. For example, in Streptomyces coelicolor, proteins involved in both primary and secondary metabolic pathways exhibit substantial post-translational modifications (Hesketh et al., 2002). Future quantitative analyses of protein abundances (for example, enabled by stable isotope labeling; Eng and Mann, 2005) in both

Table 1 Numbers of aligned variant proteins determined to be more abundant than orthologous proteins encoded by the backbone composite $A$. phosphatis genome according to functional categories

Functional category $\begin{gathered}\text { Number } \\ \text { (percentage) }\end{gathered}$

Phosphate transport and metabolism

Polyhydroxyalkanoate metabolism

$5(1.2)$

Fatty acid metabolism

TCA/glyoxylate cycle

$4(1.0)$

Carbohydrate metabolism

$30(7.2)$

$30(7.2)$

$33(8.0)$

Nitrogen metabolism

Energy production and conversion

$6(1.4)$

$28(6.8)$

Transport

$30(7.2)$

$23(5.6)$

Signal transduction mechanism

$59(14.3)$

Amino acid metabolism

Translation, ribosomal structure and biogenesis

$17(4.1)$

Post-translational modification, protein

$23(5.6)$

turnover, chaperones

Transcription

$6(1.4)$

DNA/RNA processing, replication,

$25(6.0)$

recombination and repair

Nucleotide metabolism

$1(0.2)$

Cell cycle control, cell division, chromosome

$8(1.9)$ partitioning

Intracellular trafficking, secretion and vesicular

$2(0.5)$

transport

Flagellum structure and biosynthesis

$2(0.5)$

$4(1.0)$

$9(2.2)$

Defense mechanisms

General function

$39(9.4)$

Unknown function

$30(7.2)$

Percentages of more abundant variant proteins belonging to the different functional categories provided in brackets.
Figure 3 Identification of sludge protein variants. (a) Examples of relative protein abundances for key metabolic enzymes arranged in genome order (from top to bottom) that exhibit substantial differences between the backbone (BB) composite $A$. phosphatis genome and aligned orthologous sequences from USJ, USP and OZP. Black elements indicate aligned orthologous proteins that were not identified and grey elements indicate absence of aligned orthologous proteins. Malate synthase highlighted in red. (b) Alignment of amino acid and corresponding nucleotide sequences (arranged by codon sequence from top to bottom) of orthologous malate synthases from the US and OZ sludges. Predicted amino acid sequence and amplified nucleotide sequence of the dominant UK malate synthase included in the bottom row for comparison. Peptide sequences detected using the LTQ-Orbitrap highlighted in green, predicted UK peptides are shaded in grey. Amino acid substitutions are highlighted in red and non-synonymous single nucleotide polymorphisms highlighted in blue. 
phases and detection of post-translationally modified proteins (Tyers and Mann, 2003) will more definitively resolve these issues.

$\mathrm{P}_{\mathrm{i}}$ transformations underpin the pre-eminent role of the A. phosphatis population in EBPR. Multiple mechanisms for polyP degradation and energy generation may be employed during the anaerobic phase (Figure 2a). Exopolyphosphatase (PPX) and polyphosphate kinase 2 (PPK2) both degrade polyP. PPX produces $\mathrm{P}_{\mathrm{i}}$, which in turn allows the maintenance of the proton motive force either through proton transport pyrophosphatase or by export via a low affinity $\mathrm{P}_{\mathrm{i}}$ transporter and, hence, production of ATP via $\mathrm{F}_{0} \mathrm{~F}_{1}$-type ATP synthases. In contrast, PPK2 produces GTP. A high abundance of identified GTPases within the proteomic data suggests an essential role for GTP as an energy transfer molecule within the $A$. phosphatis population. However, this correlation requires future experimental validation. Although polyphosphate kinase 1 (PPK1) obtained from an $A$. phosphatis-dominated community was previously found to be unable to hydrolyze polyP (McMahon et al., 2002), an A. phosphatis ortholog was only identified in the anaerobic protein extract (Supplementary Table 6), suggesting a possible involvement of PPK1 in anaerobic polyP degradation.

In the EBPR metabolic model, it is proposed that polyphosphate AMP phosphotransferase hydrolyses polyP to produce ADP, which in turn is converted to ATP by adenylate kinase (Figure 2a). This cycle can be reversed during the aerobic phase, allowing the replenishment of polyp (Figure 2b). Simultaneously, PPK1 may produce polyP at the expense of ATP in the aerobic phase and PPK2 may produce polyP at the expense of GTP. Excess GDP may inhibit PPX via production of guanosine bisdiphosphate (ppGpp) and guanosine pentaphosphate (pppGpp), resulting in excess polyP accumulation (Kornberg et al., 1999). PolyP synthesis requires uptake of extracellular $\mathrm{P}_{\mathrm{i}}$ via low (PIT) and high (PST) affinity transporters. Given the relatively high levels of $\mathrm{P}_{\mathrm{i}}$ in EBPR, it may be assumed that most $\mathrm{P}_{\mathrm{i}}$ translocation occurs through PIT, as described earlier (García Martín et al., 2006). Interestingly, a potential PIT was more abundant in the anaerobic phase, whereas another was solely identified in the aerobic phase (Figure 2 and Supplementary Table 6). This raises the possibility that different specialized PIT transporters may be active in the anaerobic and aerobic phases. Furthermore, the unexpected high abundance of PST in the anaerobic phase may indicate $P_{i}$ efflux via PST during the anaerobic phase, possibly coupled to ATP generation (Figure 2a).

The anaerobic carbon metabolism of EBPR has attracted considerable attention. Short-chain fatty acids (acetate in our case) are rapidly assimilated during the anaerobic phase. According to protein abundances, acetate is preferentially activated via the high affinity enzyme acetyl-CoA synthase (Figure 2a). Glycogenolysis and glycolysis provide reducing equivalents $[\mathrm{NAD}(\mathrm{P}) \mathrm{H}]$ for the subsequent polymerization of acetyl-CoA to PHAs (Figure 2a). The PHA content of EBPR biomass generally exceeds theoretical levels obtained through provision of $\mathrm{NAD}(\mathrm{P}) \mathrm{H}$ via glycogen catabolism alone (Schuler and Jenkins, 2003; Seviour et al., 2003). Additional sources have been suggested and include the anaerobic operation of the TCA (Pereira et al., 1996) and/or glyoxylate cycle (Louie et al., 2000). However, García Martín et al. (2006) highlighted the requirement for anaerobic reoxidation of reduced quinones produced by succinate dehydrogenase in the absence of electron acceptors, and proposed that this may be achieved by a novel cytochrome $b / b 6$. From our proteomic data, we are unable to verify this hypothesis, as this cytochrome was not identified. Instead, we suggest the following four alternative metabolic processes for the flux of reducing equivalents during the anaerobic phase:

(a) Fatty acid $\beta$ oxidation: Proteins involved in fatty acid degradation and synthesis were identified and found to be highly abundant. An $A$. phosphatis acyl-CoA dehydrogenase (involved in the first enzymatic step of fatty acid $\beta$ oxidation) was only identified in the anaerobic protein extract whereas an acetyl-CoA carboxylase (first enzymatic step of fatty acid biosynthesis) was only abundant in the aerobic phase (Supplementary Table 6). Furthermore, the identified enzyme enoyl-CoA hydratase (PhaJ) directly links fatty acid $\beta$ oxidation to PHA formation (Figure 2a). Consequently, apart from the requirement of fatty acids for lipid biosynthesis, $\beta$ oxidation of fatty acids stored during the aerobic phase may provide reducing equivalents for PHA formation in the anaerobic phase as suggested previously (Wilmes et al., 2008).

(b) Denitrification and operation of full TCA/glyoxylate cycle: Laboratory-scale SBRs dominated by A. phosphatis have shown ability to denitrify (Zeng et al., 2003). Although the majority of enzymes involved in denitrification were encoded by the composite A. phosphatis genome, García Martín et al. (2006) lamented the absence of a respiratory nitrate reductase (NAR) due to the lack of quinol reductase subunit (napC) in the NAR operon. In addition to NAR subunits (napDAGHB), we found a napC homologue elsewhere in the composite genome, which was identified by proteomics. A similar disjointed NAR operon structure has been reported previously in Shewanella putrefaciens, which is a denitrifier (Richardson et al., 2001). Several subunits of NAR and subunits of other enzymes involved in denitrification were identified by proteomics (Figure 2a). Consequently, we infer an active denitrification pathway in $A$. phosphatis, which enables the operation of the TCA and/ or glyoxylate cycle when nitrate is present in the anaerobic phase (Figure 2a). 
(c) Anaerobic respiration via a split TCA cycle: Fumarate reductase was identified. This enzyme enables the operation of a split TCA cycle, providing propionyl-CoA moieties for inclusion into PHAs (Figure 2a).

(d) Glyoxylate shunt: Isocitrate lyase and malate synthase were identified possibly facilitating a partial TCA cycle during the anaerobic phase (Figure 2a).

From nuclear magnetic resonance studies, the Entner-Doudoroff (ED) pathway had been considered to be the main glycolytic pathway employed by A. phosphatis (Hesselmann et al., 2000). However, all the enzymes for the Embden-Meyerhof (EM) pathway are present on the composite genome (García Martín et al., 2006) and were identified by proteomics (Figure 2). In contrast, only one putative ED specific enzyme, 6-phosphogluconate dehydratase, was identified. Combined metabolomic and proteomic studies are required to conclusively demonstrate the importance of the EM pathway.

EPS produced by sludge-dwelling organisms are essential for floc formation and, hence, retention of cells within the EBPR cycle. EPS biosynthesis cassettes within A. phosphatis were found to differ between the US and OZ sludges (García Martín et al., 2006). Based on the anomalously high degree of variability among EPS biosynthesis modules, it is not surprising that we did not identify EPS biosynthesis proteins described by García Martín et al. (2006) in the UK sludge. The possible EPS biosynthesis proteins we identified indicate that the extracellular polymers in our reactor would mainly be constituted of glucose, galactose and rhamnose sugars (Figure 2b).

A most striking finding from the proteogenomic analysis is the differential detection of orthologous proteins from closely related $A$. phosphatis variants (Figure 3a and Supplementary Figure 3). Reactors operating under subtly different conditions should enrich for particular protein variants, but overall ecosystem heterogeneity will retain genetic diversity. A large proportion of variant proteins involved in EBPR-specific biochemical processes as well as 'housekeeping' functions were more abundant than those encoded by the $A$. phosphatis composite genome (Table 1 and Supplementary Table 5). Such variation likely alters the kinetics and specificity of enzymes and may provide a means for the adaptation of distinct strains to specific environmental conditions. As illustrated by malate synthase, the relatively high abundance of a particular variant in the UK sludge is likely due to conditions that favored growth of an A. phosphatis strain that was only a minor constituent in the US sludge (Figure 3b).

Strain variation within the $A$. phosphatis population may be selectively neutral. However, a more likely explanation is that genes specific to one strain convey distinct functionality within the population.
Niche partitioning and genetic adaptation are essential for selection of organisms along specific nutrient gradients (Johnson et al., 2006). Within activated sludge, microbial flocs harbour numerous micro-niches for the dominant population and flanking organisms. Furthermore, significant gradients have been previously described in wastewater bioreactors (Schramm et al., 1999). The proteomic data suggest the coexistence of functionally distinct strain variants, which may be important for community optimization in the heterogeneous EBPR system. Interestingly, these findings are analogous to results of a macroecological study in which $\mathrm{P}_{\mathrm{i}}$ removal was enhanced in wetland mesocosms with higher macrophyte diversity (Engelhardt and Ritchie, 2002). Furthermore, increases in microbial diversity have been shown to increase the level of ecosystem functioning (Bell et al., 2005).

Our results suggest that proteins differing by only a few amino acids may be functionally distinct. Hence, proteogenomic investigations of complex microbial systems require resolution at the strain level (Lo et al., 2007). Unfortunately, genomic coverage of all constituent members within complex microbial communities is unrealistic. However, the large-scale implementation of MS-based top-down and bottom-up de novo sequencing (Steen and Mann, 2004) will diminish the requirement for comprehensive genomic foundations, and allow strain-resolved analysis of even more complex microbial ecosystems in the future.

The percentage of proteins encoded by A. phosphatis and identified in the current study was lower than that obtained in the only previous comprehensive community proteogenomic study, which focused on an acid mine drainage (AMD) biofilm dominated by Leptospirillum group II (Ram et al., 2005). However, wastewater microbial communities are more complex than AMD biofilms, and the composite A. phosphatis genome (5842 ORFs) is about double the size of the Leptospirillum group II genome (2862 ORFs). In addition, in the current study, the two genomic datasets and the proteomics samples came from wastewater reactors from three different continents (in the AMD study, the proteomics samples and genomic datasets came from the same general site). Furthermore, no distinct cellular fractions were prepared (the AMD study included extensive cellular fractionation). Despite these additional challenges, the results were comprehensive enough to provide new insights into the biochemistry of EBPR. Importantly, such insights may provide clues to optimize consortia and maintain stable EBPR operation. For example, the apparent importance of fatty acid synthesis and degradation may provide an $A$. phosphatis enrichment strategy based on pre-fermentation of exogenous fatty acids prior to the anaerobic/aerobic sludge cycling. This fermentation process could be engineered to produce volatile fatty acids with longer chain lengths than acetate. These in turn would require less 
energy expenditure for the accumulation of intracellular macromolecules (polyhydroxyalkanoate and fatty acids) and provide additional selective pressure for PAOs. Indeed, propionate has been found to be a more favourable substrate for EBPR compared to acetate in laboratory-scale reactors (Oehmen et al., 2005). Furthermore, the apparent ability of the $A$. phosphatis population to denitrify may lead to the addition of anoxic zones to current EBPR biological wastewater treatment plants that solely employ anaerobic/aerobic phases. Again, this should lead to an enrichment of the A. phosphatis population and result in better phosphate removal performance.

The present study demonstrates the power of proteogenomic methodologies when applied to complex microbial consortia that are of fundamental biotechnological and environmental importance. The results reveal an intriguing level of fine-scale genetic variation among closely related organisms and, consequently, highlight the likely importance of strain variation for overall community homeostasis.

\section{Acknowledgements}

We thank Phil Hugenholtz, Héctor García Martín and Ernest Szeto for provision of the genomic information. Furthermore, we thank Vincent Denef, Greg Dick and Sheri Simmons for helpful comments and discussions. Funding was provided by the United States Department of Energy Genomics:GTL Program (Office of Science). AA was supported by a grant from the Swedish Research Council.

\section{References}

Abe T, Sugawara H, Kinouchi M, Kanaya S, Ikemura T. (2005). Novel phylogenetic studies of genomic sequence fragments derived from uncultured microbe mixtures in environmental and clinical samples. DNA Res. 12: 281-290.

Altschul SF, Madden TL, Schäffer AA, Zhang J, Zhang Z, Miller W et al. (1997). Gapped BLAST and PSI-BLAST: a new generation of protein database search programs. Nucleic Acids Res 25: 3389-3402.

Bell T, Newman JA, Silverman BW, Turner SL, Lilley AK. (2005). The contribution of species richness and composition to bacterial services. Nature 436: 1157-1160.

Benjamini Y, Hochberg Y. (1995). Controlling the false discovery rate: a practical and powerful approach to multiple testing. J R Statist Soc B 57: 289-300.

Blackall LL, Crocetti GR, Saunders AM, Bond PL. (2002). A review and update of the microbiology of enhanced biological phosphorus removal in wastewater treatment plants. Antonie van Leeuwenhoek 81: 681-691.

Bond PL, Hugenholtz P, Keller J, Blackall LL. (1995). Bacterial community structures of phosphate-removing and non-phosphate-removing activated sludges from sequencing batch reactor. Appl Environ Microbiol 61: 1910-1916.

Correll DL. (1998). The role of phosphorus in the eutrophication of receiving waters: a review. J Environ Qual 27: 261-266.

Crocetti GR, Banfield JF, Keller J, Bond PL, Blackall LL. (2002). Glycogen-accumulating organisms in laboratory-scale and full-scale wastewater treatment process. Microbiology 148: 3353-3364.

Crocetti GR, Hugenholtz P, Bond PL, Schuler A, Keller J, Jenkins D et al. (2000). Identification of polyphosphate-accumulating organisms and design of $16 \mathrm{~S}$ rRNA-directed probes for their detection and quantification. Appl Environ Microbiol 66: 1175-1182.

Denef VJ, Shah MB, VerBerkmoes NC, Hettich RL, Banfield JF. (2007). Implications of strain- and species-level sequence divergence for community and isolate shotgun proteomic analysis. J Proteome Res 6: 3152-3161.

Eng JK, McCormack AL, Yates III JR. (1994). An approach to correlate tandem mass spectral data of peptides with amino acid sequences in a protein database. $J \mathrm{Am}$ Soc Mass Spectrom 5: 976-989.

Eng S-E, Mann M. (2005). Mass spectrometry-based proteomics turn quantitative. Nat Chem Biol 1: 252-262.

Engelhardt KAM, Ritchie ME. (2002). The effect of aquatic plant species richness on wetland ecosystem processes. Ecology 83: 2911-2924.

Florens L, Carozza MJ, Swanson SK, Fournier M, Coleman MK, Workman JL et al. (2006). Analysing chromatin remodeling complexes using shotgun proteomics and normalized spectral abundance factors. Methods 40: 303-311.

García Martín H, Ivanova N, Kunin V, Warnecke F, Barry KW, McHardy AC et al. (2006). Metagenomic analysis of two enhanced biological phosphorus removal (EBPR) sludge communities. Nat Biotechnol 24: 1263-1269.

He S, Gall DL, McMahon KD. (2007). "Candidatus Accumulibacter" population structure in enhanced biological phosphorus removal sludges as revealed by polyphosphate kinase genes. Appl Environ Microbiol 73: $5865-5874$.

Hesketh AR, Chandra G, Shaw AD, Rowland JJ, Kell DB, Bibb MJ et al. (2002). Primary and secondary metabolism, and post-translational protein modifications, as portrayed by proteomic analysis of Streptomyces coelicolor. Mol Microbiol 46: 917-932.

Hesselmann RPX, Von Rummell R, Resnick SM, Hany R, Zehnder AJB. (2000). Anaerobic metabolism of bacteria performing enhanced biological phosphate removal. Water Res 34: 3487-3494.

Hesselmann RPX, Werlen C, Hahn D, van der Meer JR, Zehnder AJB. (1999). Enrichment, phylogenetic analysis and detection of a bacterium that performs enhanced biological phosphate removal in activated sludge. Syst Appl Microbiol 22: 454-465.

Johnson ZI, Zinser ER, Coe A, McNulty NP, Woodward EMS, Chisholm SW. (2006). Niche partitioning among Prochlorococcus ecotypes along ocean-scale environmental gradients. Science 311: 1737-1739.

Kohonen T. (1990). The self-organizing map. Proc IEEE 78: 1464-1480.

Kong Y, Nielsen JL, Nielsen PH. (2004). Microautoradiographic study of Rhodocyclus-related polyphosphateaccumulating bacteria in full-scale enhanced biologi- 
cal phosphorus removal plants. Appl Environ Microbiol 70: 5383-5390.

Kong Y, Xia Y, Nielsen JL, Nielsen PH. (2005). Ecophysiology of a group of uncultured gammaproteobacterial glycogen-accumulating organisms in full-scale enhanced biological phosphorus removal wastewater treatment plants. Environ Microbiol 8: 479-489.

Kornberg A, Rao NN, Ault-Riche D. (1999). Inorganic polyphosphate: a molecule of many functions. Annu Rev of Biochem 68: 89-125.

Lo I, Denef VJ, VerBerkmoes NC, Shah MB, Goltsman D, DiBartolo G et al. (2007). Strain-resolved community proteomics reveals recombining genomes of acidophilic bacteria. Nature 446: 537-541.

Louie TM, Mah TJ, Oldham W, Ramey WD. (2000). Use of metabolic inhibitors and gas chromatography/mass spectrometry to study poly- $\beta$-hydroxyalkanoates metabolism involving cryptic nutrients in enhanced biological phosphorus removal systems. Water Res 34: 1507-1514.

Ludwig W, Strunk O, Westram R, Richter L, Meier H, Yadhukumar et al. (2004). ARB: a software environment for sequence data. Nucleic Acids Res 32: 13631371.

Markowitz VM, Korzeniewski F, Palaniappan K, Szeto E, Werner G, Padki A et al. (2006). The integrated microbial genomes (IMG) system. Nucleic Acids Res 34: D344-D348.

McHardy AC, García Martín H, Tsirigos A, Hugenholtz P, Rigoutsos I. (2007). Accurate phylogenetic classification of variable-length DNA fragments. Nat Methods 4: 63-72.

McMahon KD, Dojka MA, Pace NR, Jenkins D, Keasling JD. (2002). Polyphosphate kinase from activated sludge performing enhanced biological phosphorus removal. Appl Environ Microbiol 68: 4971-4978.

Mino T, van Loosdrecht MCM, Heijnen JJ. (1998). Microbiology and biochemistry of the enhanced biological phosphate removal process. Water Res 32: 3193-3207.

Oehmen A, Yuan Z, Blackall LL, Keller J. (2005). Comparison of acetate and propionate uptake by polyphosphate accumulating organisms and glycogen accumulating organisms. Biotechnol Bioeng 91: 162-168.

Pereira H, Lemos PC, Reis MAM, Crespo JPSG, Carrondo MJT, Santos H. (1996). Model for carbon metabolism in biological phosphorus removal processes based on in vivo ${ }^{13} \mathrm{C}-\mathrm{NMR}$ labelling experiments. Water Res 30: 2128-2138.

Ram RJ, VerBerkmoes NC, Thelen MP, Tyson GW, Baker BJ, Blake II RC et al. (2005). Community proteomics of a natural microbial biofilm. Science 308: 1915-1920.

Richardson DJ, Berks BC, Russell DA, Spiro S, Taylor CJ. (2001). Functional, biochemical and genetic diversity of prokaryotic nitrate reductases. Cell Mol Life Sci 58: 165-178.
Schramm A, De Beer D, Van den Heuvel JC, Ottengraf S, Amann R. (1999). Microscale distribution of populations and activities of Nitrosospira and Nitrospira spp. along a macroscale gradient in a nitrifying bioreactor: quantification by in situ hybridization and the use of microsensors. Appl Environ Microbiol 65: 3690-3696.

Schuler AJ, Jenkins D. (2003). Enhanced biological phosphorus removal from wastewater by biomass with different phosphorus contents, Part III: Anaerobic sources of reducing equivalents. Water Environ Res 75: 512-522.

Seviour RJ, Mino T, Onuki M. (2003). The microbiology of biological phosphorus removal in activated sludge systems. FEMS Microbiol Rev 27: 99-127.

Srinath EG, Sastry CA, Pillai SC. (1959). Rapid removal of phosphorus from sewage by activated sludge. Experientia 15: 339-340.

Steen H, Mann M. (2004). The ABC's (and XYZ's) of peptide sequencing. Nat Rev Mol Cell Biol 5: 699-711.

Tabb DL, McDonald WH, Yates III JR. (2002). DTASelect and contrast: tools for assembling and comparing protein identifications from shotgun proteomics. J Proteome Res 1: 21-26.

Tyers M, Mann M. (2003). From genomics to proteomics. Nat Biotechnol 422: 193-197.

Ultsch A, Moerchen F. (2005). ESOM-Maps: tools for clustering, visualization, and classification with Emergent SOM. Technical Report of the Department of Mathematics and Computer Science, University of Marburg, Germany 46: 1-7.

United Nations World Water Assessment Programme (2006). The United Nations World Water Development Report 2. United Nations Educational, Scientific and Cultural Organization (UNESCO) and Berghahn Books: Paris and New York, France and USA.

White D. (2007). The Physiology and Biochemistry Of Prokaryotes. Oxford University Press: New York, USA.

Wilmes P, Bond PL. (2004). The application of twodimensional polyacrylamide gel electrophoresis and downstream analyses to a mixed community of prokaryotic microoganisms. Environ Microbiol 6: 911-920.

Wilmes P, Bond PL. (2006a). Metaproteomics: studying functional gene expression in microbial ecosystems. Trends Microbiol 14: 92-97.

Wilmes P, Bond PL. (2006b). Towards exposure of elusive metabolic mixed-culture processes: the application of metaproteomic analyses to activated sludge. Water Sci Technol 54: 217-226.

Wilmes P, Wexler M, Bond PL. (2008). Metaproteomics provides functional insight into activated sludge wastewater treatment. PLoS ONE 3: 1-11 e1778.doi:10.1371/journal.pone.0001778.

Zeng RJ, Saunders AM, Yuan Z, Blackall LL, Keller J. (2003). Identification and comparison of aerobic and denitrifying polyphosphate-accumulating organisms. Biotechnol Bioeng 83: 140-148.

Supplementary Information accompanies the paper on The ISME Journal website (http://www.nature.com/ismej) 